\title{
Microvascular density as an independent predictor of clinical outcome in renal cell carcinoma: an automated image analysis study
}

\author{
Vladimir V lakovlev ${ }^{1,2}$, Manal Gabril ${ }^{3}$, William Dubinski ${ }^{1,2}$, Andreas Scorilas ${ }^{4}$, Youssef M Youssef ${ }^{2}$, Hala Faragalla ${ }^{1,2}$, \\ Kalman Kovacs ${ }^{1}$, Fabio Rotondo ${ }^{1}$, Shereen Metias ${ }^{1}$, Androu Arsanious ${ }^{1}$, Anna Plotkin ${ }^{1,2}$, Andrew HF Girgis ${ }^{1}$, \\ Catherine J Streutker ${ }^{1,2}$ and George M Yousef ${ }^{1,2}$
}

Tumor microvascular density (MVD) has been shown to correlate with the aggressiveness of several cancers. With the introduction of targeted anti-angiogenic therapy, assessment of MVD has the potential not only as a prognostic but also as a therapeutic marker. The significance of tumor vascularity in clear cell renal cell carcinoma (ccRCC) has been debated, with studies showing contradictory results. Previous studies were limited by manual quantification of MVD within a small area of tumor. Since then, the validity of this method has been questioned. To avoid the inaccuracies of manual quantification, we employed a computerized image analysis, which allowed assessment of large areas of tumor and adjacent normal tissue. The latter was used as an internal reference for normalization. MVD and vascular endothelial growth factor (VEGF) were assessed in 57 cases of ccRCC. Sections were immunostained for CD34 and VEGF. Areas of cCRCC and normal kidney medulla were analyzed within scanned images using software that counted CD34-positive vessels and measured the intensity of VEGF staining. We obtained unadjusted values from tumoral areas and calculated adjusted values as tumor/normal ratios. Unadjusted MVD had no association with clinical outcome. However, similarly to tumor stage, higher adjusted MVD was associated with shorter disease-free survival (log-rank $P=0.037, \operatorname{Cox} P=0.02$ ). This was significant in univariate and multivariate analyses. MVD did not correlate with tumor stage, pointing to its independent prognostic value. As expected due to the known molecular abnormalities in ccRCC, most tumors showed higher VEGF expression than normal tissue. Higher adjusted VEGF was associated with high tumor grade $(P=0.049)$. The finding of increased MVD as an independent marker of tumor aggressiveness may prove useful in the development of new tests for prognostic and therapeutic guidance. Digital techniques can provide more accurate assessment of immunomarkers and may reveal less obvious associations.

Laboratory Investigation (2012) 92, 46-56; doi:10.1038/labinvest.2011.153; published online 31 October 2011

KEYWORDS: CD34; image analysis; kidney cancer; microvascular density; renal cell carcinoma; tumor vascularity; VEGF

Clear cell renal cell carcinoma (ccRCC) represents the most common subtype of adult kidney cancer. ${ }^{1}$ Although early diagnosis and treatment of the disease is associated with a favorable prognosis, patients diagnosed at the metastatic stage have abysmal prospects, with only a $9 \% 5$-year survival rate. ${ }^{2}$ Neoplasms resected at a pre-metastatic stage can also recur, with the greatest risk of recurrence in the first 3-5 years. ${ }^{3}$ Detecting recurrences early can impact patient outcome since the likelihood of a good response to systemic treatment is greater when the metastatic burden is small. ${ }^{4}$ In addition, surgical resection of a single or limited number of metastases can result in longer survival. ${ }^{5}$ Furthermore, $\sim 3 \%$ of patients will develop a second primary renal tumor, either synchronously or metachronously. ${ }^{6}$

The strongest validated prognostic factors in RCC are stage (anatomic extent of disease) and nuclear grade. ${ }^{7}$ These parameters are, unfortunately, not very reliable and from a clinical standpoint, ccRCC is notoriously unpredictable.

\footnotetext{
'Department of Laboratory Medicine and Pathobiology, University of Toronto, Toronto, ON, Canada; ${ }^{2}$ Department of Laboratory Medicine, Keenan Research Centre, Li Ka Shing Knowledge Institute, St Michael's Hospital, Toronto, ON, Canada; ${ }^{3}$ Discipline of Pathology, London Health Sciences Centre, London, ON, Canada and ${ }^{4}$ Department of Biochemistry and Molecular Biology, Faculty of Biology, University of Athens, Panepistimiopolis, Athens, Greece

Correspondence: Dr GM Yousef, MD, PhD, FRCPC (Path), MSC, MBBCh, Department of Laboratory Medicine, St Michael's Hospital, 30 Bond Street, Toronto, ON M5B 1W8, Canada.

E-mail: yousefg@smh.ca

Received 29 October 2010; revised 27 July 2011; accepted 1 September 2011
} 
Reports have documented the metastatic potential of even low grade, early stage ccRCC. At the same time, only a portion of advanced stage tumors result in metastatic disease. To address these difficulties, a prognostic model utilizing multiple factors has been developed at Memorial Sloan-Kettering based on a multivariate analysis of patients with metastatic disease. ${ }^{8}$ This model has had further enhancements based on data from the Cleveland Clinic. ${ }^{9}$

Recently, moving into the era of personalized medicine, new markers have shown promise in predicting prognosis in ccRCC, including B7H1, B7H $4^{10}$ IMP3 $^{11}$ and CAIX. ${ }^{12}$ Molecular profiling approaches have also identified new biomarkers. ${ }^{13-18}$ However, none has gained widespread acceptance as a clinical tool. The identification of additional independent prognostic markers is urgently needed to help in more accurately identifying aggressive ccRCC, as this will have a significant impact on treatment protocols in the current era of targeted therapy. The assessment of microvascular density (MVD) may prove useful in this endeavor and has several advantages as a clinical test, being simple and relatively quick to assess, while using widely available techniques like immunohistochemistry (IHC) on formalin-fixed paraffin-embedded tissues. Currently, digital imaging technology allows for more reproducible automated analysis of IHC results.

The assessment of tumor MVD has been reported as a prognostic indicator in several human malignancies. Specifically, studies have shown that higher tumor vascularity correlates with worse patient outcomes in breast, ${ }^{19-21}$ prostate $^{22}$ and ovarian cancers. ${ }^{23}$ To date, studies assessing the prognostic value of MVD in ccRCC have shown conflicting results. ${ }^{24-31}$ Maclennan and Bostwick ${ }^{25}$ and Sandlund et al ${ }^{29}$ both concluded that the extent of angiogenesis has no prognostic significance in relation to patient survival. On the other hand, Yoshino et $a l^{31}$ and Nativ et $a l^{27}$ found that increased MVD was a poor prognostic factor associated with shorter survival. In Yoshino et al's ${ }^{31}$ case, higher MVD was also correlated with a higher metastatic rate. In contrast to the above studies, Imao $e t a^{24}$ and Yildiz et $a l^{30}$ showed that increased MVD in RCC correlated with longer patient survival. These contradictions are not unique to kidney tumors and one of the apparent reasons is the methodology of MVD assessment. An early and popular method of assessing MVD used 'hot-spots,' based on manually counting vessels in the most vascularized areas of histological sections. Originally, it was used in breast cancer and showed a direct relationship between MVD and tumor aggressiveness. Many subsequent studies confirmed these results. However, a substantial number of reports showed no relationship, or an inverse relationship between the vascularity and clinical outcome. $^{32-36}$ This discrepancy was attributed to methodological differences, distributional heterogeneity of microvasculature and biological differences between tumor types. In addition, low MVD has been reported as a poor prognostic marker in pancreatic endocrine tumors, ${ }^{37}$ bladder carcinoma $^{38,39}$ and cervical cancer. ${ }^{40}$ MVD has also been found to vary between different tumor types of the same organ. $^{41,42}$ What became apparent, was that the hot-spot methodology for determining MVD was poorly reproducible ${ }^{34,43,44}$ and several studies employed a Chalkley graticule, ${ }^{44,45}$ which improved reproducibility, but could not resolve the issue of conflicting results. ${ }^{44,46}$

The current advances in digital pathology allow MVD to be assessed with much higher expected accuracy. Computerized approaches offer the capability of analyzing much larger areas and also consistently apply analysis algorithms. Our goal was to improve the accuracy of measurements and test the old hypothesis that MVD correlates with clinical outcome.

In clinical practice, the significance of MVD in ccRCC may have a dual function: prognostic assessment and treatment guidance. Recently, a number of clinical trials have tested the effectiveness of anti-angiogenic therapy for advanced RCC. In particular, Sunitinib and Sorafenib have been approved by the FDA for the treatment of metastatic renal carcinoma. ${ }^{47,48}$ The rationale for anti-angiogenic therapy originates from studies documenting the necessity of tumor vasculogenesis for the maintenance, growth and metastatic ability of the tumor. ${ }^{49}$ However, present antiangiogenic therapies have shown only modest effects on disease progression while adding a significant cost. ${ }^{50,51}$ Detected responses were seen mainly with higher drug doses, which had higher incidences of side effects. Therefore, there is a need for a predictive marker to identify tumors likely to respond in order to justify the higher risk of toxicity and cost of treatment.

In this study, we report the first automated digital assessment of MVD in ccRCC. MVD was assessed in parallel with vascular endothelial growth factor (VEGF), a major driver of angiogenesis. ${ }^{36}$ We aimed to improve accuracy of measurements by applying strict criteria for quality of staining, tissue integrity and selection of tissue of interest. We also employed data normalization by analyzing normal tissue and calculating tumor/normal ratio, or adjusted values. The unadjusted and adjusted data were then compared for their correlation with prognostic clinical parameters to provide insight into the effect of data normalization as well as the potential use of MVD as a prognostic marker in ccRCC.

\section{MATERIALS AND METHODS \\ Tissue Collection}

With Research Ethics Board approval, 57 consecutive cases of surgically removed ccRCC's between 2001 and 2008 were obtained from the division of Pathology at St Michael's Hospital (Toronto, ON, Canada). Clinico-pathologic data were obtained from the patient medical records and included sex, age, disease-free survival (DFS), overall survival, tumor size, TNM stage and nuclear grade. As per routine reporting protocol, RCC tumors were graded based on the Fuhrman four-scale grading system ${ }^{52}$ and staged according to the 
American Joint Committee on Cancer (AJCC) 7th edition. Hematoxylin-eosin sections were reviewed by pathologists (GMY and VVI) to reconfirm the diagnosis and grading and to select appropriate paraffin blocks for IHC.

\section{Immunohistochemistry}

All specimens had been fixed in 10\% neutral-buffered formalin, dehydrated in graded ethanol and paraffin embedded. Sections of $4 \mu \mathrm{m}$ thickness were stained with hematoxylin and eosin, as well as CD34 and VEGF. The primary antibodies anti-CD34 (pre-diluted; Ventana Medical Systems, Tucson, AZ, USA) and VEGF (1:50; Santa Cruz Biotechnology, Santa Cruz, CA, USA) were used for staining on a Benchmark staining platform (Ventana Medical Systems), with the BMK iVIEW DAB Paraffin detection kit (Ventana Medical Systems). Heat antigen retrieval was used for VEGF antibody: kit cell conditioner \#1 (Ventana Medical Systems) with heating to $95^{\circ} \mathrm{C}$ for $8 \mathrm{~min}$, followed by $20 \mathrm{~min}$ at $100^{\circ} \mathrm{C}$. I-VIEW inhibitor was incubated for $4 \mathrm{~min}$ at $42{ }^{\circ} \mathrm{C}$. For CD34 antigen retrieval, protease 2 was used for $10 \mathrm{~min}$ incubation. All slides were then incubated with primary antibody for $32 \mathrm{~min}$, followed by I-VIEW biotin immunoglobulin for 8 min, I-VIEW SA-HRP for 8 min, I-VIEW DAB and $\mathrm{H}_{2} \mathrm{O}_{2}$ for $8 \mathrm{~min}$ and I-VIEW copper for $4 \mathrm{~min}$. Slides were counterstained with hematoxylin and bluing reagent. After drying, slides were scanned using ScanScope CS (Aperio Technologies, CA, USA) at $\times 20$ magnification and saved as an eightbit RGB TIFF file at $2 \mu \mathrm{m} /$ pixel resolution.

\section{Image Analysis}

Image analysis was performed similarly to previously described methods. ${ }^{53}$ The initial image processing of generating signal-mask pairs was achieved using 'Photoshop CS3' (Adobe, http://www.adobe.com). The scanned images (representative areas are shown in Figure 1) were duplicated into 'tumor' and 'normal' folders and manually processed to remove all parts of the image except either tumor or normal kidney medulla within the images of corresponding folders. All areas of necrosis, hemorrhage or non-specific background staining, which could be removed by the eraser tool, were excluded from assessment. Additionally, for normal tissue, only areas without significant changes (fibrosis, inflammation etc) were selected for analysis. In cases where no useable normal tissue was present, only unadjusted values from the tumor areas were recorded.

Within both folders, the images were processed to generate two sets of files: tissue masks to limit analysis within the selected areas (tumor or medulla) and signal images to assess the markers. The tissue masks were obtained by thresholding through a value separating the clear background of histological slides from the tissue. The procedure generated binary black-and-white images. The sequence of transformations was recorded as an 'action' (Photoshop, Adobe) and images were processed in batches to save as bitmap files. Next, original images were processed to generate signal images of
CD34 and VEGF brown stain. Since CD34 signal was used to count objects, its brown color was converted into solid white color for binary bitmap images (Figure 2), whereas VEGF was assessed as intensity and so its signal was converted into grayscale TIFFs. For CD34, the range of positive brown pixels was collected to convert into black using the 'convert color' tool and then thresholding to generate binary images. Both conversion and thresholding steps were adjusted to achieve selection of vascular structures without fragmentation or fusion of vessels. We de-noised the images by 1 pixel using the 'dust and scratches' filter (Figure 2). The sequence of transformations was recorded as an 'action,' tested on a representative set of images to assure the correct settings and used for batch processing. Then images were checked for quality and fragmented large vessels were additionally restored manually. The vascular objects were counted as vascular density (vessel $/ \mathrm{mm}^{2}$ ) using an image analysis program based on language IDL 6.3 (ITT Visual Information Solutions, Boulder, CO, USA), which has been used in earlier studies. ${ }^{53-55}$ The VEGF set of images was processed similarly in batches, but instead of thresholding, the brown color was converted into the grayscale signal using a previously described CMYK approach. ${ }^{56}$ The grayscale VEGF signal was measured as intensity of staining (mean optical density, MOD) within the tissue masks.

\section{Statistical Analysis}

The MVD and VEGF data were collected for both tumor and normal kidney medulla and tumor/normal ratios, or 'adjusted' tumor values were calculated. Cutoff points for high and low groups were selected as follows: unadjusted MVD-median of 536.9 vessels $/ \mathrm{mm}^{2}$; adjusted MVD, 1.0 (< normal vs >normal); unadjusted VEGF-median of 40.22 MOD, adjusted VEGF, 1.0. Since the cases were split into two groups by MVD and VEGF measurements, for direct comparison, the cases were also stratified into two groups by their stage. The stages were either combined as stages 1 and $2 v s 3$, or $1 v s 2$ and 3 . By tumor grade, the cases were stratified as two groups combining I and II and III and IV grades.

Statistical analysis was performed using XLSTAT version 2010.4.02 (Addinsoft, http://www.xlstat.com). Continuous variables were compared between groups by the non-parametric two-tailed Mann-Whitney test and correlations between continuous variables were assessed by the Spearman test. We used Cox model and Kaplan-Meier analysis to assess associations with DFS. Since tumors with adjusted MVD of $<1$ had no recurrence events, the hazard ratio could not be calculated, but we were able to calculate the $P$-value of the likelihood ratio test to assess the significance of associations between the parameters and DFS.

\section{RESULTS}

The descriptive statistics of the patients involved in this study is summarized in Table 1. As described in the Materials and 

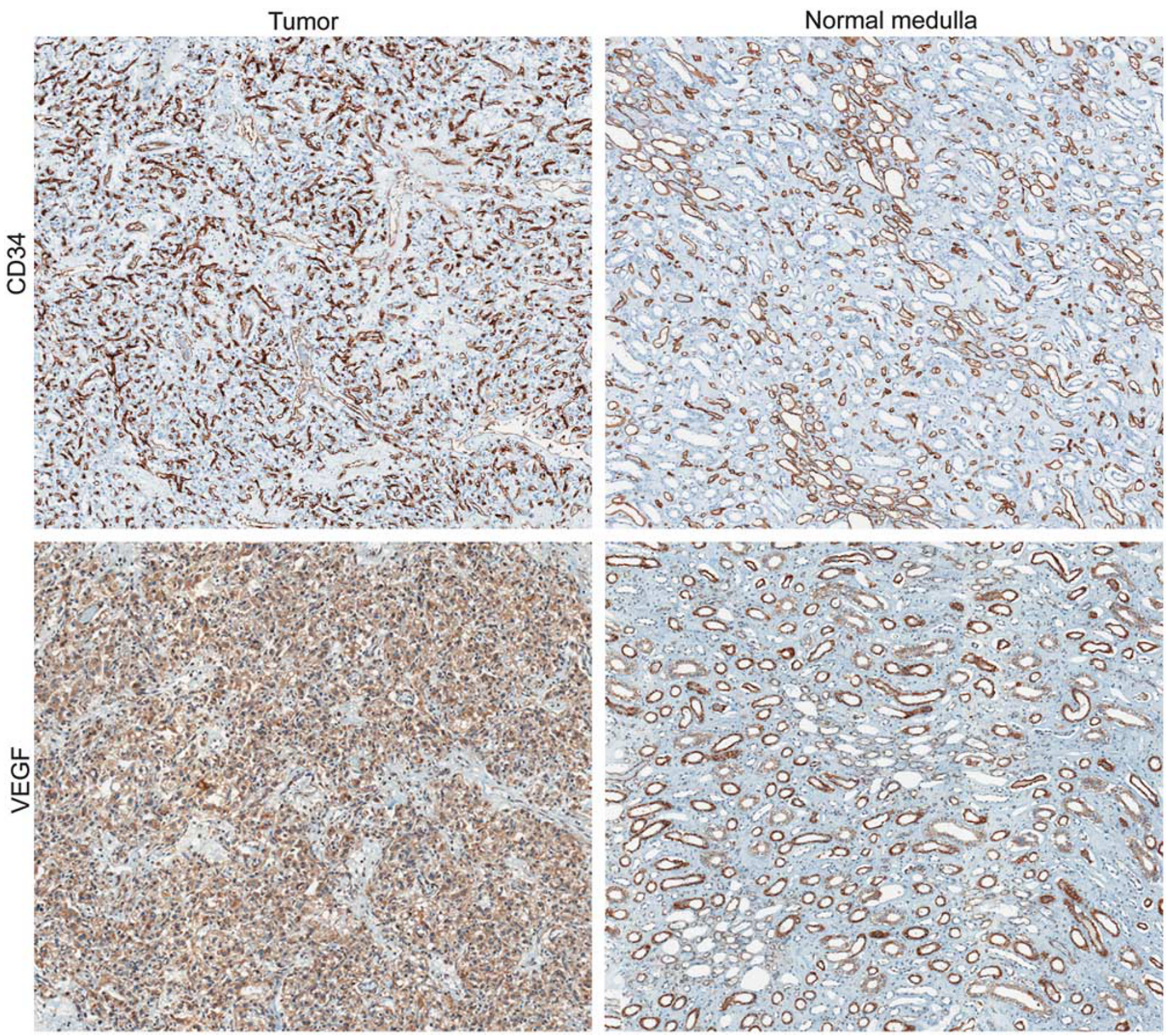

Figure 1 Representative images of CD34 and VEGF staining in tumor and normal kidney medulla.

Methods section, only technically acceptable and specific tissue areas were included in the analysis. Their number varied within the CD34 and VEGF sets.

As a preparatory step, we tested kidney cortex and medulla separately for their usability as an internal reference of MVD. Figure 1 shows representative images of CD34 and VEGF staining. Both MVD and VEGF were assessed as unadjusted and adjusted (tumor/normal ratio) values. Our analysis showed that the cortex could not be used as a normal reference because dense glomerular capillary loops could not be reliably separated into individual vascular rings (Figure 2); therefore, we used kidney medulla as an internal reference. For VEGF, we did not observe a specific advantage of either using cortex or medulla as an internal reference and used medulla for consistency.

\section{Associations with DFS}

Both tumor stage and adjusted MVD showed similar associations with DFS by Kaplan-Meier analysis (Figure 3). Higher adjusted MVD was associated with significantly shorter DFS. All cases with recurrences occurred in the tumors where MVD was higher than in the normal kidney medulla (log-rank $P=0.037$ ) (Figure 3a). Higher stages were also associated with a worse prognosis (log-rank $P=0.041$ when stages 1 and 2 were combined together, and $P=0.03$ when stages 2 and 3 were merged in one group). We also applied the Cox proportional hazards model to test the significance of associations with DFS. Higher adjusted MVD showed significant association with tumor recurrences by univariate (likelihood ratio test $P=0.02$ ) as well as multivariate analysis in combination with tumor stage (adjusted 

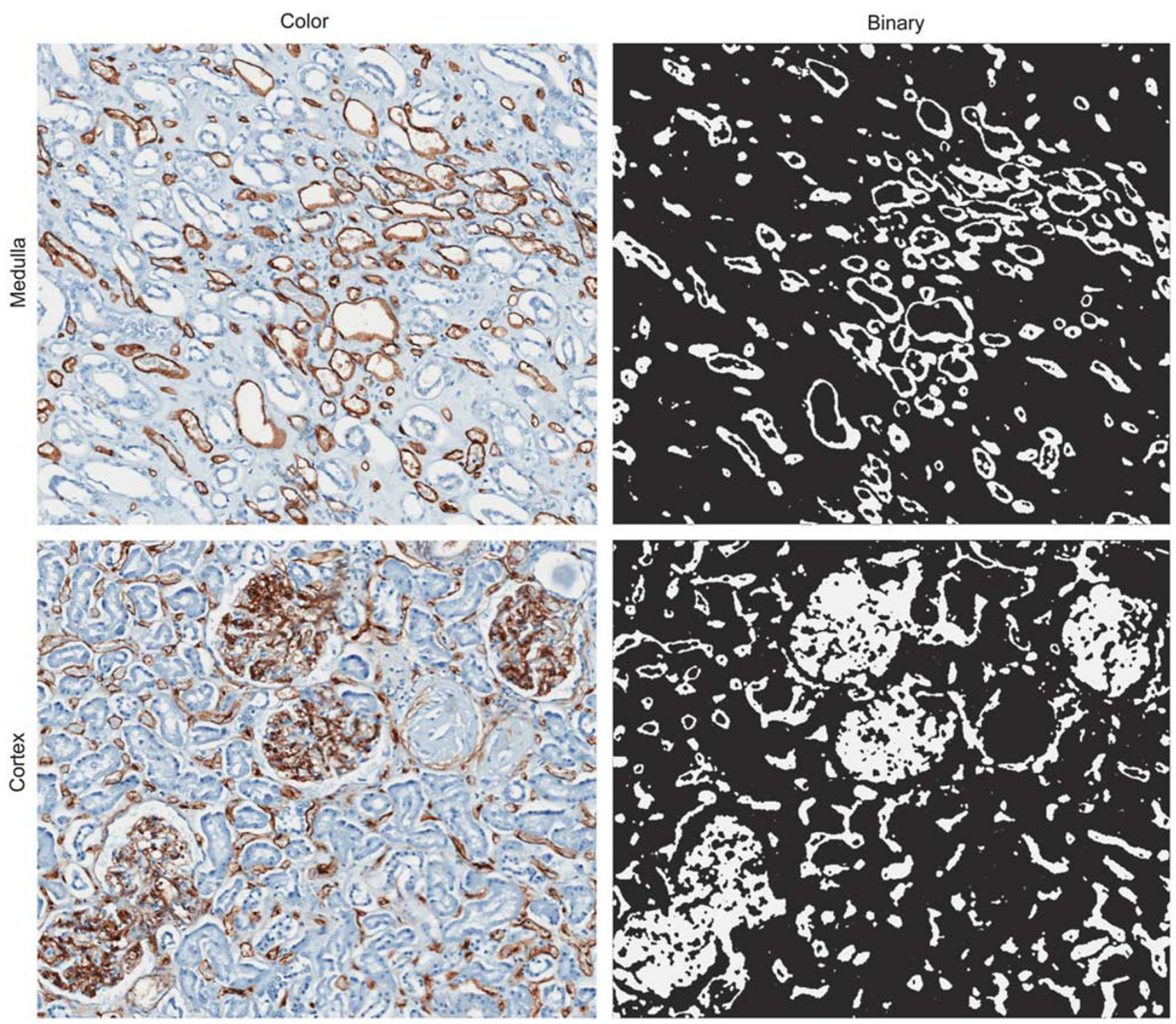

Figure 2 Selection of vascular structures by analysis algorithm within normal kidney cortex and medulla. Note that the glomeruli in the cortex with their dense capillary networks each produce a complex, however single, vascular object. Also note that the sclerotic glomerulus is an avascular scar. Variability of glomerular density between individuals and within the cortex (denser in the outer parts) did not allow us to use the renal cortex as a standardized internal control for MVD assessment.

MVD $P=0.032$ ). As expected, higher stage tumors had a higher chance to recur $(P=0.056$ when stages 1 and 2 were grouped together, and $P=0.013$ when stages 2 and 3 were grouped together; no significant difference for stage 1 vs 2 or $2 v s 3$ ). The scatter of recurrences $v s$ adjusted MVD and stage are shown in Figure 4a. Interestingly, all recurrent cases fell into the high tumor/normal MVD and stage 3 quadrant (Figure 4a). Figure $4 \mathrm{~b}$ demonstrates the distribution of recurrences in tumors of different grades. Tumor recurrence did not show an association with higher grade. All recurrent cases showed, however, tumor/normal MVD ratio $>1.0$, indicating that adjusted MVD is a better indicator for recurrence than grade. Figure $4 \mathrm{c}$ shows raw MVD data in relation to stage.
Adjusted VEGF ratio was available in two tumors, which recurred after surgery. Although this number was too low to assess the significance, both cases had an adjusted VEGF expression $>1.0$ (Figure 5a). The unadjusted VEGF data did not show an association with DFS (Figure $5 b$ ).

\section{Associations Between MVD, VEGF and Other Clinical Parameters}

An important finding was that, although both adjusted MVD and stage showed comparable associations with DFS, there was no correlation between them as either continuous (Spearman $=-0.028$; Table 2$)$ or ordinate variables (MVD medians 0.87 and 0.89 , Mann-Whitney $P=0.8$ ). Figure 4 shows that there was an approximately symmetrical 
Table 1 Descriptive statistics of the numerical variables in the study ${ }^{a}$

\begin{tabular}{|c|c|c|c|}
\hline Variable & $N$ & Median & Range \\
\hline \multicolumn{4}{|l|}{ MVD } \\
\hline Unadjusted (vessel/mm²) & 57 & 536.9 & $196.2-1376.9$ \\
\hline Ratio to normal & 46 & 0.82 & $0.31-2.10$ \\
\hline \multicolumn{4}{|l|}{ VEGF } \\
\hline Unadjusted intensity $\left(\mathrm{MOD}^{\mathrm{b}}\right)$ & 53 & 40.22 & $8.4-157.1$ \\
\hline Ratio to normal & 46 & 1.26 & $0.38-3.8$ \\
\hline Age (years) & 50 & 64.0 & $23.0-87.0$ \\
\hline \multicolumn{4}{|l|}{ Sex } \\
\hline Male & 36 & & \\
\hline Female & 21 & & \\
\hline \multicolumn{4}{|l|}{ Stage } \\
\hline 1 & 30 & & \\
\hline 2 & 9 & & \\
\hline 3 & 18 & & \\
\hline \multicolumn{4}{|l|}{ Grade } \\
\hline । & 4 & & \\
\hline$\|$ & 30 & & \\
\hline III & 19 & & \\
\hline IV & 4 & & \\
\hline Tumor size $(\mathrm{cm})$ & 57 & 5.5 & $2.5-15.0$ \\
\hline Follow-up time (months) & 57 & 39.0 & $8.0-131.0$ \\
\hline
\end{tabular}

Abbreviations: MVD, microvascular density; VEGF, vascular endothetical growth factor; MOD, mean optical density.

anly technically acceptable and specific tissue areas were included in the analysis, as described in the Materials and Methods section.

${ }^{\mathrm{b}}$ Mean optical density (intensity) of staining.

distribution of tumors with adjusted MVD values $<1$ and $>1$ in the stage groups: 14 with the MVD $<1$ and 11 with the ratio $>1$ for stage 1 , compared with 12 and 8 for stages 2 and 3 combined. Another important finding was that the raw MVD measurements, unlike the adjusted ratios, had no association with DFS. As shown in Table 2, we observed a weak trend of decreasing unadjusted MVD in larger tumors (Spearman $=0.29, P=0.04)$. However, there was no difference of unadjusted MVD between the stage groups, which reflect tumor size and the trend was not seen for adjusted MVD data.

It is well known that the VHL gene is inactivated in most RCC while VEGF expression is suppressed by VHL in normal non-hypoxic cells. In our data set, 35 out of $46(76 \%)$ tumors showed VEGF expression higher than in normal tissue (Figure 5a). Adjusted VEGF correlated moderately with tumor size (Table 2; Spearman $=0.36, P=0.014$ ) and grade (Table 2; Spearman $=0.3, P=0.039$; Table 3; Figure 5a). We observed higher adjusted VEGF in higher-grade tumors (grouped as grades I and II and III and IV, median $1.23 \mathrm{vs}$ 1.46, respectively, Mann-Whitney $P=0.049$ ). Raw VEGF a

DFS and tumor/normal MVD ratio

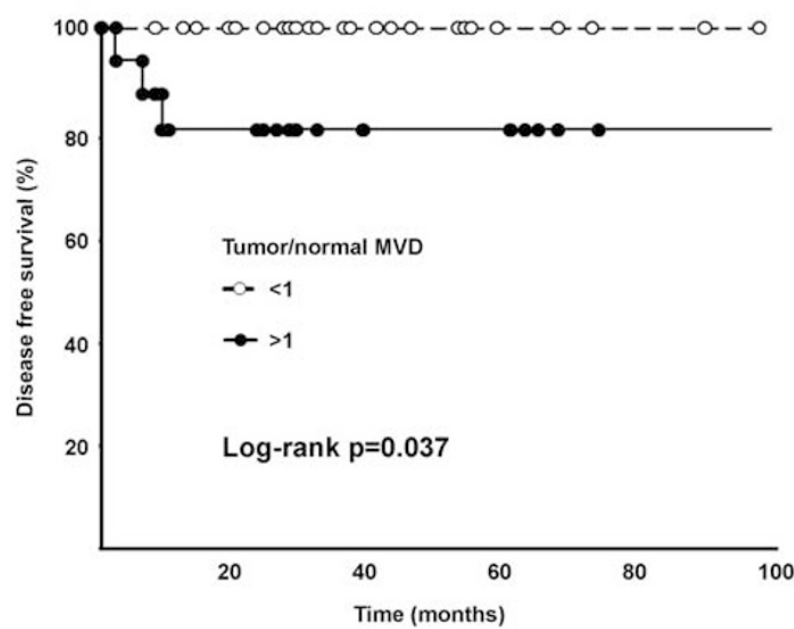

b

DFS and tumor stage (1\&2 combined)

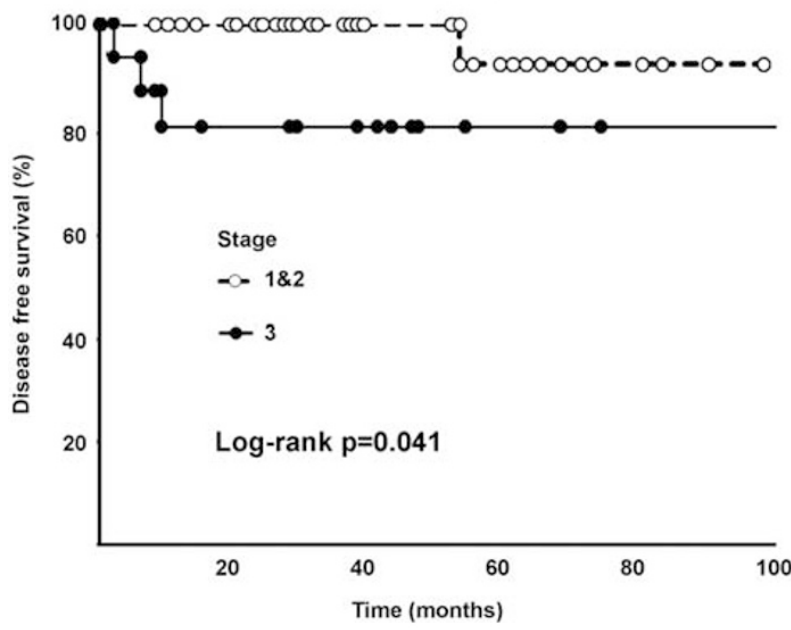

c

DFS and tumor stage (2\&3 combined)

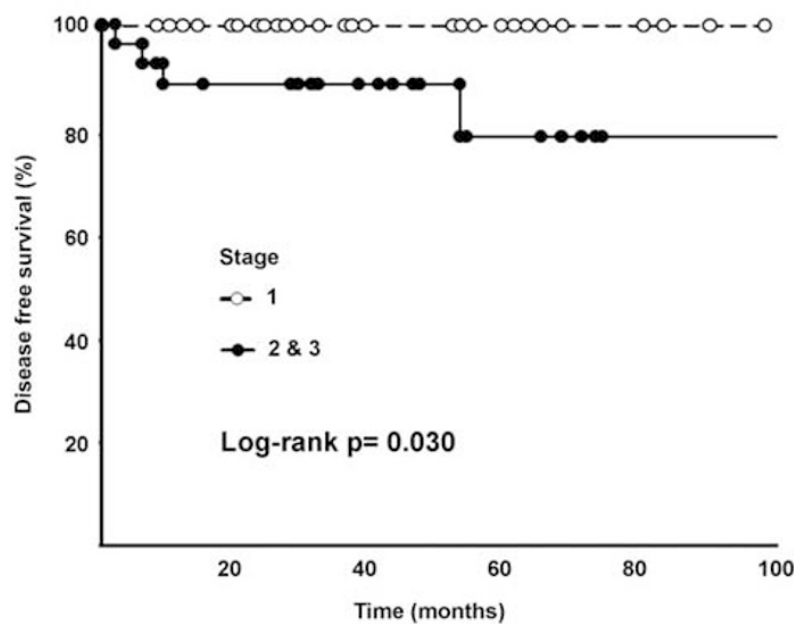

Figure 3 Kaplan-Meier plots for disease free survival (DFS) by (a) tumor/ normal MVD ratio, as assessed by CD34 staining, and (b, c) tumor stage. Tumor stage data were organized in two groups by either combining stages 1 and 2 (b) or 2 and 3 (c). 

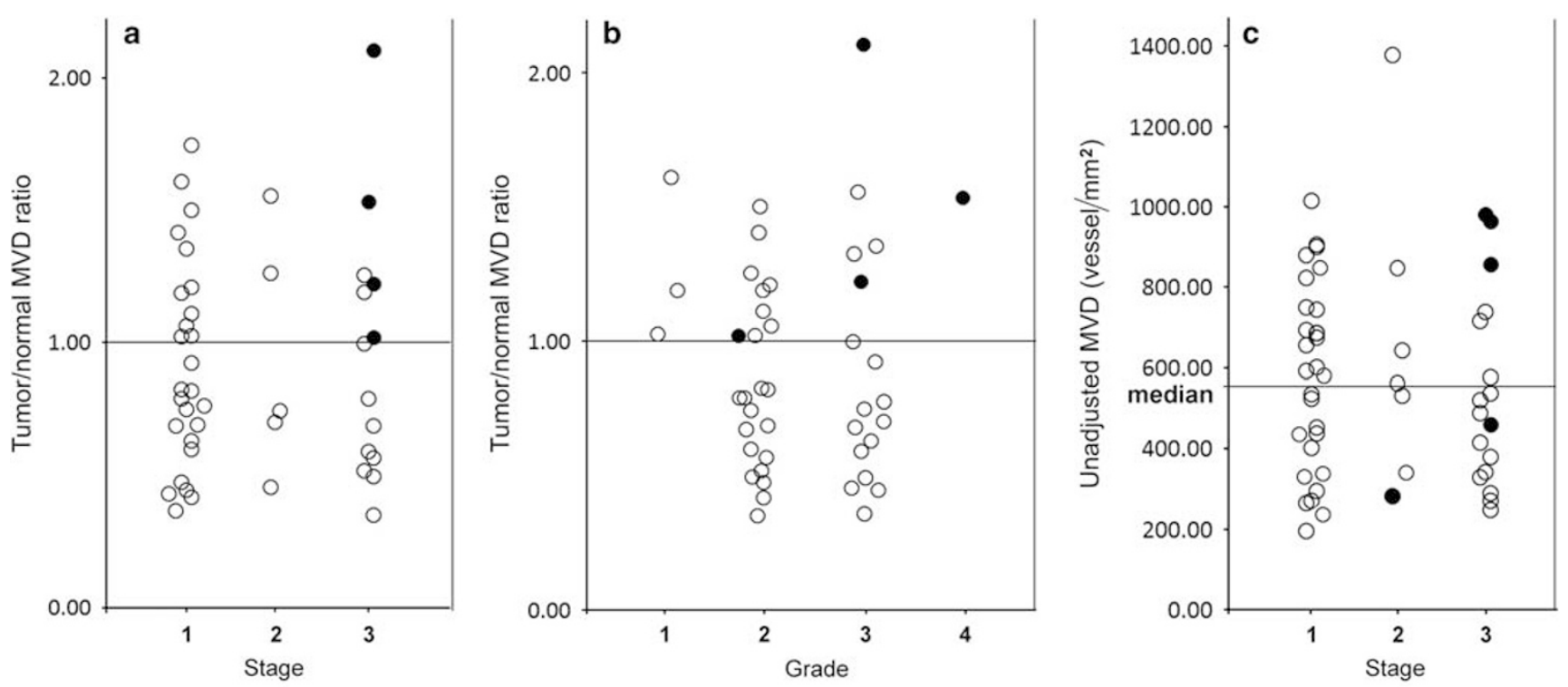

No recurrence

- Recurrence

Figure 4 Distribution of tumor recurrences vs microvascular density (MVD), stage and grade of the tumors. (a) MVD assessed by adjusted MVD/normal medulla compared with stage (b) MVD assessed by adjusted MVD/normal medulla compared with grade and (c) MVD assessed by unadjusted MVD compared with stage. Normal medulla for MVD measurements was available for four tumors with recurrence. Note that all four of these fall in the 'high stage-high tumor/normal MVD' quadrant (a).
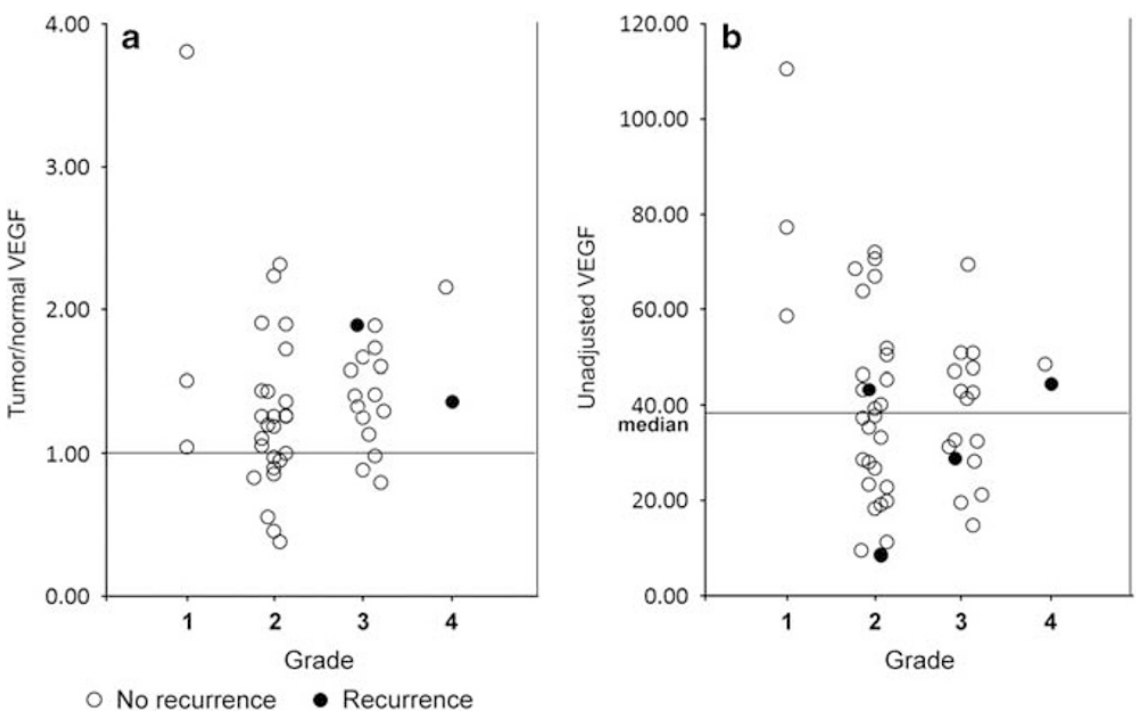

Figure 5 Vascular endothelial growth factor (VEGF) and grade data distribution. Tumor/normal VEGF ratio was available for assessment in only two tumors, both showed values higher than the median (a). Also note that most of the tumors had VEGF expression higher than normal tissue, which is consistent with the common genetic mechanism in RCC. There was a trend of higher VEGF expression in the higher-grade tumors. Unadjusted data are shown for comparison in panel b.

data did not correlate with either grade (Figure 5b), stage, MVD or other parameters (Table 3).

\section{DISCUSSION}

Our study was based on the old hypothesis that solid neoplasms require a vascular network for growth, and a rapidly growing tumor would have a higher vascularity to support the proliferation demands. Despite the fact that the topic has been studied for over 40 years, there is still no clear understanding of what the relationship is between tumor microvasculature and its aggressiveness. Since previous studies employed manual counting methods, their analyses were limited to small areas and were subject to observer-related variability. Without a gold standard, it is difficult to estimate the magnitude of the combined error, but it is likely that a large proportion of previous studies had an error too large to 
Table 2 Correlation matrix (Spearman) between the parameters used for analysis

\begin{tabular}{|c|c|c|c|c|c|c|c|}
\hline Variables & Tumor size $(\mathrm{cm})$ & pT stage & Grade & MVD raw & MVD t/n & VEGF raw & VEGF $\mathrm{t} / \mathrm{n}$ \\
\hline Tumor size $(\mathrm{cm})$ & 1 & & & & & & \\
\hline pT stage & $0.559^{\mathrm{a}}$ & 1 & & & & & \\
\hline Grade & 0.460 & 0.002 & 1 & & & & \\
\hline MVD raw ${ }^{b}$ & -0.289 & -0.236 & -0.099 & 1 & & & \\
\hline$M V D t / n^{c}$ & -0.035 & -0.281 & -0.007 & 0.834 & 1 & & \\
\hline VEGF raw & -0.114 & -0.078 & 0.043 & -0.103 & -0.118 & 1 & \\
\hline VEGF $\mathrm{t} / \mathrm{n}$ & 0.359 & 0.028 & 0.305 & -0.032 & 0.189 & 0.003 & 1 \\
\hline
\end{tabular}

Abbreviations: MVD, microvascular density; VEGF, vascular endothetical growth factor; $\mathrm{t} / \mathrm{n}$, tumor/normal.

${ }^{\mathrm{a}}$ Values in bold are different from 0 with a significance level $\alpha<0.05$.

${ }^{b}$ Raw $=$ unadjusted.

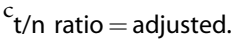

Table 3 MVD and VEGF data vs tumor stage and grade

\begin{tabular}{|c|c|c|c|c|c|}
\hline & \multicolumn{2}{|c|}{ MVD } & \multicolumn{2}{|c|}{ VEGF } & \\
\hline & Raw $^{\mathrm{a}}$ (vessel/mm²) & $\mathrm{t} / \mathrm{n}^{\mathrm{b}}$ ratio & Raw (mean optical density) & $\mathrm{t} / \mathrm{n}$ ratio & \\
\hline \multicolumn{6}{|c|}{ Stage } \\
\hline 1 & $642(232-1377) N=29$ & $0.83(0.31-1.75) N=25$ & $41(9-83) N=29$ & $1.26(0.56-2.24) N=26$ & \\
\hline 2 & $331(196-880) N=9$ & $0.75(0.42-1.61) N=6$ & $40(15-111) N=7$ & $1.90(1.0-3.8) N=6$ & \\
\hline 3 & $469(248-980) N=17$ & $0.89(0.35-2.10) N=14$ & $37(8-72) N=15$ & $1.26(0.38-2.16) N=14$ & \\
\hline \multicolumn{6}{|c|}{ Grade } \\
\hline 1 & $750(744-823) N=3$ & $1.19(1.03-1.61) N=3$ & $59(38-77) N=4$ & $1.01(0.85-1.91) N=4$ & \multirow{2}{*}{] $1.23^{c}$} \\
\hline$\|$ & $532(248-1015) N=29$ & $0.79(0.35-1.5) N=23$ & $35(8-83) N=27$ & $1.26(0.38-2.32) N=24$ & \\
\hline III & $438(196-1377) N=14$ & $0.70(0.31-2.1) N=17$ & $42(15-111) N=18$ & $1.49(0.87-3.8) N=16$ & \multirow{2}{*}{] $1.46^{\mathrm{C}}$} \\
\hline IV & $741(469-980) N=4$ & $1.64(1.53-1.75) N=2$ & $52(45-59) N=2$ & $1.44(1.36-1.51) N=2$ & \\
\hline
\end{tabular}

Abbreviations: MVD, microvascular density; VEGF, vascular endothetical growth factor; t/n, tumor/normal.

${ }^{\mathrm{a}}$ Raw $=$ unadjusted.

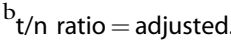

${ }^{c}$ Values in bold are different from 0 with a significance level $\alpha<0.05$.

The difference of either MVD or VEGF between individual stage and grade groups was not significant. Combining smaller groups (stage 2 , grades 1 and 4 ) with the adjacent larger groups showed that adjusted VEGF values were significantly different between combined grades I and II and III and IV (Mann-Whitney $P=0.049$ ).

resolve any true biological differences. For example, in hypoxia studies, distribution of the hypoxia marker carbonic anhydrase $I X$ was shown to be heterogeneous by immunohistochemical measurements and $3 \mathrm{D}$ reconstruction. ${ }^{53,57}$ Only extensive tumor sampling revealed its correlation with tissue oxygen tension measured in vivo. Noticeably, the degree of correlation was increasing with the increasing amount of tumor tissue sampled for immunohistochemical analysis.

Our approach was to minimize both technical and sampling errors. The latest computerized imaging techniques allow for analysis of large areas, which reduces the sampling error caused by tumor heterogeneity. In addition, computer analysis algorithms are consistent, which reduces analytical error. We also aimed to address the recently raised issue of variable antigen preservation within archived tissues, which can have an effect on sensitivity of staining. The time lag between surgery and fixation, size of the specimen and overall fixation time are known to have an effect on tissue preservation. Other factors, such as storage time of paraffin blocks, tissue water content and variability of section thickness can also have an effect on intensity of staining. Practically speaking, it is difficult or impossible to control for all of these factors. These variables directly affect measurements of staining intensity (type of analyses similar to VEGF assessment) and can alter analyses based on object counting (like MVD). Even for the latter type of image analysis, the filters still have a threshold to exclude non-specific background staining. The same filter setting can produce a different 
number of individual objects due to either fusion of adjacent bright objects or fragmentation of objects with pale staining. Setting a filter manually for each image would introduce an operator-dependent variability. Instead, we employed an internal reference to normalize the data. Since both the tumor and normal tissue within the same section are exposed to the same fixation variables, their ratio is expected to be affected minimally. Due to the inherent even distribution of vessels, we used kidney medulla as an internal reference and calculated tumor/normal ratios, or 'adjusted' values.

Using adjusted MVD, we observed an association between high vascular density and reduced DFS (Figure 3). The association was comparable to that of the established prognostic factor, tumor stage. Importantly, only the adjusted MVD, but not the raw MVD measurements, showed a correlation with poorer prognosis. Adjusted MVD and stage were independently associated with decreased DFS, and all tumors with recurrences fell into the 'high MVD-high stage' quadrant of the distribution (Figure 4). As seen in Figure 4, the data suggest that, for clinical purposes, adjusted MVD measurements may have a role in selecting more aggressive tumors within the higher-stage groups. A possible biological explanation could be that the more aggressive tumors drive angiogenesis, which either supports or stimulates tumor cell proliferation and invasiveness. The more indolent tumors reach similar size and stage slowly exhausting their vascular supply.

VEGF is the best-characterized mediator of endothelial sprouting, the most common method by which tumors acquire new vascular channels. ${ }^{58}$ In the presence of VEGF, endothelial cell survival is enhanced and helps to form the vascular network enabling tumor metastases. ${ }^{58,59}$ Inclusion of normal tissue into the analysis allowed us to compare the levels of VEGF expression in tumors and normal tissue. Due to the common loss of VHL gene function, the expression level of the normally suppressed VEGF, was expected to be higher in RCCs. ${ }^{60}$ In fact, in 35 out of 46 cases we did observe VEGF expression higher in tumors compared with normal tissues (76\%; Figure 5a). Also, adjusted VEGF levels were higher in higher-grade tumors, which were not observed for unadjusted values. We did not detect any correlation between VEGF expression and tumor stage or MVD. The reason for these results is likely multifactorial. Although VEGF is a major factor in angiogenesis, tumors utilize numerous other mechanisms to develop new vasculature, some of which do not rely on VEGF. ${ }^{58}$ In addition, it has been shown that VEGF levels are variable within a tumor, with hypoxic areas of tumor secreting higher levels of VEGF compared with well-vascularized areas. ${ }^{61}$ The degree of VEGF expression in tumors is likely a combination of levels predetermined by the molecular abnormalities with superimposed VEGF production as a physiological response to hypoxia. Since the latter component has an inverse relationship with the microvasculature, it can distort the association between the inherent VEGF expression levels and MVD.
Over several decades, the assessment of tumor microvasculature has drawn attention as a prognostic marker; however a large number of studies produced equivocal results. ${ }^{21,24,26,28,62-66}$ Practically, all studies were based on manual counting of vessels within small areas of tumor utilizing either 'hot-spots' ${ }^{26,30,67}$ or small tissue microarray cores. ${ }^{68}$ Both factors, small tissue sample and manual input have a large potential for errors; it is logical to replace those methods with standardized computer analyses of large areas. Moreover, the inclusion of an internal reference point is easily achieved by the new methodology and can further increase the accuracy of measurements. It can be argued that the normal tissue adjacent to the tumor can be affected by it. For example, blood flow can be shunted and the normal tissue may react by an increase in VEGF production and higher MVD. In practice, we observed the associations only using the adjusted data. Therefore, the effect is either not present or smaller than the benefit of normalization and we believe that inclusion of an internal reference has a large potential for immunohistochemical analyses in general.

Both researchers and clinicians should fully understand that accurate assessment of biomarkers is extremely important in the era of targeted therapies. The accumulated experience of currently used targeted medications shows that only a subset of patients benefit from the treatment. The result has been a thorough standardization of clinical tests and development of quality assurance programs for the laboratories performing them. Similarly, researchers should aim to use discovery tools of sufficient accuracy to resolve any potential biological differences. In this study, we aimed to increase the accuracy of immunohistochemical assessment of MVD and VEGF and our main finding was that higher adjusted MVD in ccRCC is predictive of increased tumor aggressiveness. This may prove useful for prognostic and treatment purposes in patients with ccRCC.

\section{ACKNOWLEDGEMENTS}

We thank Andrew Morrison, who contributed to the image analysis software, and Dr David Hedley at whose laboratory the software was written and tested. This research was funded by the Canadian Cancer Society Grant \#20185) and the Canadian Institute of Health Research (CIHR) (Grant \#86490), the kidney foundation of Canada and the Ministry of Research and Innovation of the Government of Ontario.

\section{DISCLOSURE/CONFLICT OF INTEREST}

The authors declare no conflict of interest.

1. Srigley JR, Delahunt B. Uncommon and recently described renal carcinomas. Mod Pathol 2009;22(Suppl 2):S2-S23.

2. Weiss RH, Lin PY. Kidney cancer: identification of novel targets for therapy. Kidney Int 2006;69:224-232.

3. Lam JS, Shvarts O, Leppert JT, et al. Postoperative surveillance protocol for patients with localized and locally advanced renal cell carcinoma based on a validated prognostic nomogram and risk group stratification system. J Urol 2005;174:466-472.

4. Antonelli A, Cozzoli A, Zani D, et al. The follow-up management of non-metastatic renal cell carcinoma: definition of a surveillance protocol. BJU Int 2007;99:296-300. 
5. Hofmann HS, Neef $\mathrm{H}$, Krohe $\mathrm{K}$, et al. Prognostic factors and survival after pulmonary resection of metastatic renal cell carcinoma. Eur Urol 2005:48:77-81.

6. Klatte $\mathrm{T}$, Patard JJ, Wunderlich $\mathrm{H}$, et al. Metachronous bilateral renal cell carcinoma: risk assessment, prognosis and relevance of the primary-free interval. J Urol 2007;177:2081-2086.

7. Amin MB, Tamboli $P$, Javidan J, et al. Prognostic impact of histologic subtyping of adult renal epithelial neoplasms: an experience of 405 cases. Am J Surg Pathol 2002;26:281-291.

8. Motzer RJ, Mazumdar M, Bacik J, et al. Survival and prognostic stratification of 670 patients with advanced renal cell carcinoma. J Clin Oncol 1999;17:2530-2540.

9. Mekhail TM, Abou-Jawde RM, Boumerhi G, et al. Validation and extension of the Memorial Sloan-Kettering prognostic factors model for survival in patients with previously untreated metastatic renal cell carcinoma. J Clin Oncol 2005;23:832-841.

10. Thompson RH, Kwon ED. Significance of B7-H1 overexpression in kidney cancer. Clin Genitourin Cancer 2006;5:206-211.

11. Hoffmann NE, Sheinin $\mathrm{Y}$, Lohse CM, et al. External validation of IMP3 expression as an independent prognostic marker for metastatic progression and death for patients with clear cell renal cell carcinoma. Cancer 2008;112:1471-1479.

12. Atkins $M$, Regan $M, M c D e r m o t t ~ D$, et al. Carbonic anhydrase IX expression predicts outcome of interleukin 2 therapy for renal cancer. Clin Cancer Res 2005;11:3714-3721.

13. Arsanious A, Bjarnason GA, Yousef GM. From bench to bedside: current and future applications of molecular profiling in renal cell carcinoma. Mol Cancer 2009;8:20.

14. Chow TF, Youssef YM, Lianidou E, et al. Differential expression profiling of microRNAs and their potential involvement in renal cell carcinoma pathogenesis. Clin Biochem 2010;43:150-158.

15. Lam JS, Pantuck AJ, Belldegrun AS, et al. Protein expression profiles in renal cell carcinoma: staging, prognosis, and patient selection for clinical trials. Clin Cancer Res 2007;13(2 Pt 2):703s-708s.

16. Romaschin $A D$, Youssef $Y, C$ how TF, et al. Exploring the pathogenesis of renal cell carcinoma: pathway and bioinformatics analysis of dysregulated genes and proteins. Biol Chem 2009;390:125-135.

17. Schaefer A, Stephan C, Busch J, et al. Diagnostic, prognostic and therapeutic implications of microRNAs in urologic tumors. Nat Rev Urol 2010;7:286-297.

18. Siu KW, DeSouza LV, Scorilas A, et al. Differential protein expressions in renal cell carcinoma: new biomarker discovery by mass spectrometry. J Proteome Res 2009;8:3797-3807.

19. Bosari S, Lee AK, DeLellis RA, et al. Microvessel quantitation and prognosis in invasive breast carcinoma. Hum Pathol 1992;23: 755-761.

20. Brem S, Cotran R, Folkman J. Tumor angiogenesis: a quantitative method for histologic grading. J Natl Cancer Inst 1972;48: 347-356.

21. Weidner N, Folkman J, Pozza F, et al. Tumor angiogenesis: a new significant and independent prognostic indicator in early-stage breast carcinoma. J Natl Cancer Inst 1992:84:1875-1887.

22. Weidner N, Carroll PR, Flax J, et al. Tumor angiogenesis correlates with metastasis in invasive prostate carcinoma. Am J Pathol 1993;143: 401-409.

23. Schoell WM, Pieber $\mathrm{D}$, Reich $\mathrm{O}$, et al. Tumor angiogenesis as a prognostic factor in ovarian carcinoma: quantification of endothelial immunoreactivity by image analysis. Cancer 1997;80:2257-2262.

24. Imao T, Egawa $\mathrm{M}$, Takashima $\mathrm{H}$, et al. Inverse correlation of microvessel density with metastasis and prognosis in renal cell carcinoma. Int J Urol 2004;11:948-953.

25. Maclennan GT, Bostwick DG. Microvessel density in renal cell carcinoma: lack of prognostic significance. Urology 1995;46:27-30.

26. Minardi D, Lucarini G, Mazzucchelli R, et al. Prognostic role of Fuhrman grade and vascular endothelial growth factor in pT1a clear cell carcinoma in partial nephrectomy specimens. J Urol 2005;174(4 Pt 1): 1208-1212.

27. Nativ $O$, Sabo $E$, Reiss $A$, et al. Clinical significance of tumor angiogenesis in patients with localized renal cell carcinoma. Urology 1998;51:693-696.

28. Sabo E, Boltenko A, Sova $Y$, et al. Microscopic analysis and significance of vascular architectural complexity in renal cell carcinoma. Clin Cancer Res 2001;7:533-537.
29. Sandlund J, Hedberg Y, Bergh A, et al. Evaluation of CD31 (PECAM-1) expression using tissue microarray in patients with renal cell carcinoma. Tumour Biol 2007;28:158-164.

30. Yildiz E, Ayan S, Goze F, et al. Relation of microvessel density with microvascular invasion, metastasis and prognosis in renal cell carcinoma. BJU Int 2008;101:758-764.

31. Yoshino $S$, Kato M, Okada K. Prognostic significance of microvessel count in low stage renal cell carcinoma. Int J Urol 1995;2:156-160.

32. Ahlgren J, Risberg B, Villman $\mathrm{K}$, et al. Angiogenesis in invasive breast carcinoma-a prospective study of tumour heterogeneity. Eur J Cancer 2002;38:64-69.

33. Fox SB, Harris AL. Histological quantitation of tumour angiogenesis. APMIS 2004;112:413-430.

34. Hlatky L, Hahnfeldt P, Folkman J. Clinical application of antiangiogenic therapy: microvessel density, what it does and doesn't tell us. J Natl Cancer Inst 2002;94:883-893.

35. Lee $\mathrm{AH}$. The relationship of vascularity of the primary tumour to lymph node metastases and to distant haematogenous spread in carcinoma of the breast. Histopathology 2002;41:366-368.

36. Sharma S, Sharma MC, Sarkar C. Morphology of angiogenesis in human cancer: a conceptual overview, histoprognostic perspective and significance of neoangiogenesis. Histopathology 2005;46:481-489.

37. Couvelard A, O'Toole D, Turley $\mathrm{H}$, et al. Microvascular density and hypoxia-inducible factor pathway in pancreatic endocrine tumours: negative correlation of microvascular density and VEGF expression with tumour progression. Br J Cancer 2005;92:94-101.

38. Bartoletti R, Cai T, Nesi G, et al. Qualitative and quantitative analysis of angiogenetic factors in transitional cell bladder carcinoma: relationship with clinical course at 10 years follow-up. Oncol Rep 2005;14:251-255.

39. Hoskin PJ, Sibtain A, Daley FM, et al. The immunohistochemical assessment of hypoxia, vascularity and proliferation in bladder carcinoma. Radiother Oncol 2004;72:159-168.

40. Kaanders JH, Wijffels $\mathrm{Kl}$, Marres $\mathrm{HA}$, et al. Pimonidazole binding and tumor vascularity predict for treatment outcome in head and neck cancer. Cancer Res 2002;62:7066-7074.

41. Dagnon K, Heudes D, Bernaudin JF, et al. Computerized morphometric analysis of microvasculature in non-small cell lung carcinoma. Microvasc Res 2008;75:112-118.

42. Yuan A, Yang PC, Yu CJ, et al. Tumor angiogenesis correlates with histologic type and metastasis in non-small-cell lung cancer. Am J Respir Crit Care Med 1995;152(6 Pt 1):2157-2162.

43. Folkman J. What is the evidence that tumors are angiogenesis dependent? J Natl Cancer Inst 1990;82:4-6.

44. Trivella M, Pezzella F, Pastorino U, et al. Microvessel density as a prognostic factor in non-small-cell lung carcinoma: a meta-analysis of individual patient data. Lancet Oncol 2007;8:488-499.

45. Chalkley HW. Method for the quantitative morphologic analysis of tissues. J Natl Cancer Inst 1943;4:47-53.

46. Hansen S, Grabau DA, Rose $C$, et al. Angiogenesis in breast cancer: a comparative study of the observer variability of methods for determining microvessel density. Lab Invest 1998;78:1563-1573.

47. Motzer RJ, Rini BI, Bukowski RM, et al. Sunitinib in patients with metastatic renal cell carcinoma. JAMA 2006;295:2516-2524.

48. Ratain MJ, Eisen T, Stadler WM, et al. Phase II placebo-controlled randomized discontinuation trial of sorafenib in patients with metastatic renal cell carcinoma. J Clin Oncol 2006;24:2505-2512.

49. Kerbel RS. Tumor angiogenesis: past, present and the near future. Carcinogenesis 2000;21:505-515.

50. Stadler WM, Kuzel T, Shapiro C, et al. Multi-institutional study of the angiogenesis inhibitor TNP-470 in metastatic renal carcinoma. J Clin Oncol 1999;17:2541-2545.

51. Yang JC, Haworth L, Sherry RM, et al. A randomized trial of bevacizumab, an anti-vascular endothelial growth factor antibody, for metastatic renal cancer. N Engl J Med 2003;349:427-434.

52. Fuhrman SA, Lasky LC, Limas C. Prognostic significance of morphologic parameters in renal cell carcinoma. Am J Surg Pathol 1982;6:655-663.

53. lakovlev VV, Pintilie M, Morrison A, et al. Effect of distributional heterogeneity on the analysis of tumor hypoxia based on carbonic anhydrase IX. Lab Invest 2007;87:1206-1217.

54. Hedley D, Pintilie M, Woo J, et al. Carbonic anhydrase IX expression, hypoxia, and prognosis in patients with uterine cervical carcinomas. Clin Cancer Res 2003;9:5666-5674. 
55. Hedley D, Pintilie M, Woo J, et al. Up-regulation of the redox mediators thioredoxin and apurinic/apyrimidinic excision (APE)/Ref-1 in hypoxic microregions of invasive cervical carcinomas, mapped using multispectral, wide-field fluorescence image analysis. Am J Pathol 2004;164:557-565.

56. Pham NA, Morrison A, Schwock J, et al. Quantitative image analysis of immunohistochemical stains using a CMYK color model. Diagn Pathol 2007; 2:8.

57. Pintilie M, lakovlev V, Fyles A, et al. Heterogeneity and power in clinical biomarker studies. J Clin Oncol 2009;27:1517-1521.

58. Dome B, Hendrix MJ, Paku S, et al. Alternative vascularization mechanisms in cancer: pathology and therapeutic implications. Am J Pathol 2007;170:1-15.

59. Benjamin LE, Golijanin D, Itin A, et al. Selective ablation of immature blood vessels in established human tumors follows vascular endothelial growth factor withdrawal. J Clin Invest 1999;103. 159-165.

60. Baldewijns MM, van Vlodrop IJ, Vermeulen PB, et al. VHL and HIF signalling in renal cell carcinogenesis. J Pathol 2010;221:125-138.

61. Flaherty KT, Puzanov I. Building on a foundation of VEGF and mTOR targeted agents in renal cell carcinoma. Biochem Pharmacol 2010;80:638-646.
62. de la Taille $A$, Katz $A E$, Bagiella $E$, et al. Microvessel density as a predictor of PSA recurrence after radical prostatectomy. A comparison of CD34 and CD31. Am J Clin Pathol 2000;113:555-562.

63. Kato T, Kameoka S, Kimura T, et al. The combination of angiogenesis and blood vessel invasion as a prognostic indicator in primary breast cancer. Br J Cancer 2003;88:1900-1908.

64. Mineo TC, Ambrogi V, Baldi A, et al. Prognostic impact of VEGF, CD31 CD34, and CD105 expression and tumour vessel invasion after radical surgery for IB-IIA non-small cell lung cancer. J Clin Pathol 2004;57:591-597.

65. Qian CN, Min HQ, Liang XM, et al. Primary study of neovasculature correlating with metastatic nasopharyngeal carcinoma using computer image analysis. J Cancer Res Clin Oncol 1997;123:645-651.

66. Tanaka F, Otake Y, Yanagihara $\mathrm{K}$, et al. Evaluation of angiogenesis in non-small cell lung cancer: comparison between anti-CD34 antibody and anti-CD105 antibody. Clin Cancer Res 2001;7:3410-3415.

67. Baldewijns MM, Thijssen VL, Van den Eynden GG, et al. High-grade clear cell renal cell carcinoma has a higher angiogenic activity than low-grade renal cell carcinoma based on histomorphological quantification and qRT-PCR mRNA expression profile. Br J Cancer 2007;96:1888-1895.

68. Sandlund J, Hedberg Y, Bergh A, et al. Endoglin (CD105) expression in human renal cell carcinoma. BJU Int 2006;97:706-710. 